\title{
Analysis of Smartphone Inter- ruptions on Academic General Internal Medicine Wards
}

\section{Frequent Interruptions may cause a 'Crisis Mode' Work Climate}

Alon Vaisman'; Robert C. Wu

'Division of General Internal Medicine, Department of Medicine, University Health Network, Toronto, Ontario

\author{
Keywords \\ Communication, interruptions, smartphones, information technology
}

\section{Summary}

Introduction: Hospital-based medical services are increasingly utilizing team-based pagers and smartphones to streamline communications. However, an unintended consequence may be higher volumes of interruptions potentially leading to medical error. There is likely a level at which interruptions are excessive and cause a 'crisis mode' climate.

Methods: We retrospectively collected phone, text messaging, and email interruptions directed to hospital-assigned smartphones on eight General Internal Medicine (GIM) teams at two tertiary care centres in Toronto, Ontario from April 2013 to September 2014. We also calculated the number of times these interruptions exceeded a pre-specified threshold per hour, termed 'crisis mode', defined as at least five interruptions in 30 minutes. We analyzed the correlation between interruptions and date, site, and patient volumes.

Results: A total of 187,049 interruptions were collected over an 18-month period. Daily weekday interruptions rose sharply in the morning, peaking between $11 \mathrm{AM}$ to $12 \mathrm{PM}$ and measuring 4.8 and 3.7 mean interruptions/hour at each site, respectively. Mean daily interruptions per team totaled $46.2 \pm 3.6$ at Site 1 and $39.2 \pm 4.2$ at Site 2. The 'crisis mode' threshold was exceeded, on average, 2.3 times/day per GIM team during weekdays. In a multivariable linear regression analysis, site ( $\beta 6.43 \mathrm{Cl} 95 \% 5.44-7.42, \mathrm{p}<0.001$ ), day of the week (with Friday having the most interruptions) ( $\beta 0.481 \mathrm{Cl} 195 \% 0.236-0.730, \mathrm{p}<0.05)$ and patient census $(\beta 1.55 \mathrm{Cl} 95 \% 1.42-1.67$, $p<0.05)$ were all predictive of daily interruption volume although there was a significant interaction effect between site and patient census $(\beta-0.941 \mathrm{Cl} 95 \%-1.18--0.703, \mathrm{p}<0.05)$.

Conclusion: Interruptions were related to site-specific features, including volume, suggesting that future interventions should target the culture of individual hospitals. Excessive interruptions may have implications for patient safety especially when exceeding a maximal threshold over short periods of time.

\section{Correspondence to:}

Alon Vaisman

Department of Medicine

University Health Network

200 Elizabeth Street

Eaton Building 14-217

Toronto ON M5G 2C4

Phone: (416) 340-4059

Fax: (416) 5955826
Appl Clin Inform 2017; 8: 1-11

https://doi.org/10.4338/ACI-2016-08-RA-0130

received: August 1, 2016

accepted: November 7, 2016

published: January 4, 2017

Citation: Vaisman A, Wu RC. Analysis of smartphone interruptions on academic general internal medicine wards: Frequent interruptions may cause a 'crisis mode' work climate. Appl Clin Inform 2017; 8: 1-11 https://doi.org/10.4338/ACI-2016-08-RA-0130 Funding

Alon Vaisman is supported by the University of Toronto Eliot Phillipson Clinician Scientist Training Program 


\section{Background and Significance}

To improve communication between health professionals in hospitals, many health care teams centralize communications to one device such as a team pager or smartphone $[1,2]$. This method has led to easier communication for nurses through fewer incorrect pages and increased connectivity [3]. There has also been a trend of replacing pagers with smartphones in the last decade [4, 5]. Physicians, nurses, and other health professionals have found smartphones to be invaluable tools in communicating issues around patient care by using secure text messages, emails, and phone calls $[6,7]$. However, an unintended consequence of centralizing communication and using smartphones may be higher volume of interruptions in the form of emails, phone calls, and text messages [7]. During resident feedback sessions at our institution, a common complaint has been the sheer volume of interruptions from the team smartphone.

Previous literature has shown that interruptions can lead to higher medical error rates among health care professionals including physicians, nurses, and pharmacists [8-11]. Interruptions can also disrupt task completion, increase workload, and can lead to poor recall about the details regarding the task at hand $[12,13]$. Interruptions via smartphones have been shown to lead to distraction during transfer of patient information $[5,14]$. However, some interruptions are essential to patient care such as the notification of a clinically deteriorating patient or emergent test results $[15,16]$. Thus, interruptions can simultaneously provide timely vital information about patients but also disrupt medical staff from performing cognitively demanding tasks. Therefore, a balance between communication and work flow needs to be achieved. With excessive interruptions, clinicians may be unable to complete tasks or even resolve earlier interruptions [17]. This may contribute to perceptions of a "crisis mode" work climate [18]. Consequently, volumes of interruptions have clear implications for patient safety and healthcare delivery [19]. Prior studies on communication in the hospital setting found that interruptions by telephone land lines and pagers occurred approximately every 18 minutes [20].

\section{Objectives}

The purpose of this study was to determine the rate of interruptions from smartphones used in the care of patients on the General Internal Medicine (GIM) ward in academic hospitals.

\section{Methods}

\subsection{Design and Setting}

We conducted a retrospective study of team-based smartphone communications at two academic care sites at the University Health Network, a tertiary hospital in Toronto, Ontario. The two sites included in the study were Toronto General Hospital (Site 1) and Toronto Western Hospital (Site 2), each with approximately 80 General Internal Medicine beds, divided into four Clinical Teaching Unit (CTU) GIM teams for a total of 8 CTU teams being studied. CTU teams staffing is routine and usually staffed by one senior second-year medical resident, three junior first-year medical residents, and one or two medical students.

Each CTU team at each of the two sites was previously provided with an institutional smartphone to receive and place phone calls, and receive and send text messages and emails. Healthcare professionals can send secure text messages to a team smartphone through the hospitals' secure communication system, Clinical Messenger. While the primary source of communication comes from nursing staff, other sources include patient flow coordinators, attending physicians, physicians from consulting services, and other health professionals. The precise source of each communication was not documented, and neither was the content of each communication, which were all presumed to be related to direct patient care, as these devices are prohibited for personal use.

We defined the term interruptions as an intrusion by an unplanned and unscheduled task, and included emails, phone calls, and text messages sent only to the team smartphone. These communi- 
cations were deemed "interruptive" for three reasons. First, all three types of communications elicited a perceptible noise/vibration when received and therefore provide a noticeable sensory interruption to the resident carrying the smartphone. Second, the individual responding to the sensory interruption must then shift his or her attention to deal with the smartphone message or phone call, leading to a temporal and cognitive interruption in completing the task at hand. Third, nearly all communications from nursing staff were not elicited and are therefore unexpected in nature causing an interruption to the receiver of the communication. Fourth, even when the recipient is engaged in using the smartphone, a new communication was deemed an interruption because it necessitated a transfer of attention from one communication to another. Thus, it is unlikely that a recipient is focused on the precise issue of the incoming communication at the time it is received and therefore the communication is interruptive. Therefore, although vital information may be delivered via one of these aforementioned communications, we deemed them interruptive because each communication necessitates a deviation of attention away from a concurrent undertaking.

The team smartphone is typically carried by the most senior resident of the team at any given time and is rarely carried by staff physicians. They are required to be on 24 hours a day to receive any urgent communication regarding patient care. Communications directed at personal devices were not tracked due to concern of privacy and feasibility. Personal pagers were generally not used in lieu of team smartphones and therefore constitute a significant minority of all communications, and therefore were not included in the study. Furthermore, although communication regarding patient care can be sent through personal pagers and personal handheld devices (although discouraged due to patient confidentiality issues), most communications are targeted to the team smartphone. As well, the model of directing communications to a single device is a common and widely used method of streamlining communication in North American tertiary centres $[1,2]$.

\subsection{Definition of Crisis Mode}

Resident feedback at our site provided information about instances when the team smartphone would repeatedly receive interruptions in a short period of time - whether by phone calls, text messages, or emails. Such interruptions prevented residents from completing work and they would be harried to respond to interruptions. We determined a threshold of excessiveness for the number interruptions per time period, a concept not previously documented in the literature regarding communication in health care. We termed this threshold, 'crisis mode, a concept applied from work climates in which staff members are "doing too many things too quickly" $[15,18]$. We determined this threshold by randomly querying 20 residents and 10 staff working on the CTUs at both sites. With face-to-face surveys, we posed the following question, "Beyond what limit of interruptions per hour, defined as phone calls, text messages, and emails, would impede your ability to complete daily clinical tasks in a safe and timely manner?" We also asked for qualitative descriptions to support their selection of the perceived 'crisis mode' value, including queries about their experiences on the wards. Based on the data provided by these surveys, we averaged the values and defined the perceived crisis mode threshold as a period of time in which there were at least 5 interruptions within 30 minutes or less, or one every 6 minutes. Using this baseline definition, we also performed a sensitivity analysis using 4 and 6 interruptions per 30 minutes for comparison.

\subsection{Data Collection}

Three types of smartphone interruptions were analyzed: clinical text messages, emails, and incoming phone calls. We collected this data for eight GIM teams from April 1, 2013 to September 30, 2014, for a total of 18 months. During this period, we also collected data regarding daily patient volume from our hospital database, which was defined as the total daily patient census for each site.

Data on the exact date and time of the clinical text messages and phone calls were obtained from the telecommunications provider. Duration of phone calls was not documented due limitations in available data. Outgoing calls, emails, and texts were not collected, as these were initiated by residents and therefore not deemed interruptions. Data on the exact date and time of incoming email communications were obtained electronically from the hospital email servers. 


\subsection{Analysis}

For the 18-month study period, we determined the frequency of each type of interruption and plotted it against time of day. The frequency of 'crisis mode' events was calculated by identifying individual occurrences throughout the study period in which 5 or more interruptions were received by a team within 30 minutes, and dividing the frequency over a 24 -hour period. This value gave us the chances of achieving the crisis mode on any given day per team. Because our definition of crisis mode was novel and not validated, we performed a sensitivity analysis on its parameters using thresholds of $20 \%$ above and below the mentioned definition. Multiple linear regression analysis was performed to identify a correlation between the number of daily interruptions per team and the following predictors: month (coded as a linear term, 1-12), day of the week (coded as a linear term), daily team patient census, and hospital site. We also test for an interaction term between site and volume. This analysis excluded the observation period during the winter holiday period (December 21 - January 2) due to changes in team structure and communication. Statistics were performed using Stata 14.1 (Lakewood, TX).

\section{Results}

A total of 187,049 interruptions were collected over an 18-month period for the eight CTU teams at the two hospital sites ( Table 1). Of these, 44,795 (23.9\%) interruptions were phone calls, 39,044 (20.9\%) were emails, and the remaining 103,210 (52.2\%) were clinical text messages. Of the total number of interruptions, 101,279 (54.1\%) were from Site 1 and the remainder from Site 2 (85 770, 45.9\%). Average daily census at each site was 19.6 patients/team at Site 1 and 19.4 patients/team at Site 2. Maximum census occurred during September 2014, at 21.0 patients/team at Site 1 and 23.3 patients/team at Site 2 .

- Figure 1 displays the average daily weekday interruptions at Site 1 and Site 2. Daily weekday interruptions at both sites rose sharply in the morning with a peak between 11AM-12PM, measuring 4.8 and 3.7 average interruptions at Site 1 and Site 2, respectively. An additional peak at 2PM-3PM yielded 4.3 and 3.0 average interruptions at Site 1 and Site 2, respectively. At Site 1, the average daily phone call, email, and clinical message interruptions were 13.3, 15.8, and 22.4, respectively, for a total of 51.4 daily weekday interruptions per CTU team. At Site 2, these values were 9.2, 6.0, and 27.1, with a daily total of 42.3 interruptions per CTU team.

Across both sites, interruptions rose over the course of the 18 months of the study, with peaks in July of both academic years. Monthly fluctuations at both sites were seen, with nadirs occurring in February 2014 for Site 2 and September 2013 for Site 1.

\subsection{Periods of excessive interruptions}

Across the 18-month study period, we determined that the excessive interruptions threshold was exceeded $2.3(\mathrm{SD}=2.0)$ times per team during weekdays and 1.0 times per team on a weekend day. Over the course of the day, the highest likelihood of surpassing the threshold was at 11:00 AM, when this exceeded a $25 \%$ chance ( Figure 2 ). The mean duration that a crisis mode would last was 35.1 minutes, with a median of 30 minutes (interquartile range 25.5 - 42.0 minutes). The maximum sustained excessive interruption period was 180.9 minutes with 36 interruptions. The highest frequency of interruptions was 57 within a 93.4-minute period.

A sensitivity analysis was conducted on the excessive interruptions threshold using $20 \%$ limits. With a lower boundary of 4.0 interruptions per 30 minutes, this threshold was exceeded 3.7 (SD = 2.2) times per day. With an upper boundary of 6.0 interruptions per 30 minutes, the threshold was exceeded $1.4(\mathrm{SD}=1.6)$ times per day. Similar temporal peaks were seen using these definitions as well. 


\subsection{Predictors of Interruptions}

The multiple linear regression analysis yielded a statistically significant $[\mathrm{F}(4,3991)=183.59, \mathrm{p}<$ 0.001 ; R2 $=0.187$ ] model predictive of our outcome. A main effect was found with three predictor variables: site ( $\beta 6.43$ CI 95\% $5.44-7.42, \mathrm{p}<0.001$ ), day of the week (with Friday having the most interruptions) ( $\beta 0.481 \mathrm{CI} 95 \% 0.236-0.730, \mathrm{p}<0.05)$ and patient volume $(\beta 1.55 \mathrm{CI} 95 \% 1.42-1.67$, $\mathrm{p}<0.05)$. However, in a model including an interaction term between site and volume, we found a significant effect from the interaction $(\beta-0.941 \mathrm{CI} 95 \%-1.18--0.703, \mathrm{p}<0.05)$. There was no main effect of month $(\beta-0.160$ CI $95 \%-0.329-0.010 \mathrm{p}=0.08)$ on the number of interruptions.

\section{Discussion}

Our findings show that there are a significant number of daily smartphone interruptions to physicians on a typical clinical teaching unit. On weekdays, teams experienced the 'crisis mode' 2.3 times per day in which their interruptions exceeded 5 events per 30 minutes. We saw a high number of interruptions on average during regular workday hours, including during 'protected' educational times between 8-9 am and 12-1 pm, times dedicated for residents to receive regularly scheduled teaching when interdisciplinary staff are instructed not to contact residents with routine ward issues [21]. The daily variation in interruption volume may be related to nursing work flow processes such as medication administration timing and handover of patient care. Monthly variations in interruption volumes, for example the peak observed in July, may be due to familiarity of residents with hospital systems and ordering processes. Interruptions were also significantly higher in one site and thus may be related to practice culture, including the education of nurses and other health professions (although this association was mediated by patient volume). Alternative explanations for the difference between the two sites may be an unmeasured variable such as complexity of patients or different numbers of patients bed-spaced to surgical wards.

In this study, we defined communications from nurses to physicians via text, email or phone calls as interruptions. It is important to recognize that communication is vital in patient care, but there is likely a balance between the importance of delivered information and the volume of communications. While more communication can be seen as a positive as it improves team coordination, there is a tension in which too much communication can be overwhelming on an individual level. This can lead to the experience of fatigue and cause individuals to ignore or delaying responses to communications [22]. Centralizing the communication to a team device likely increases the chance of this occurring.

Our study found a higher total number of interruptions compared to previous studies on interruptions on general medical wards [7, 20, 23, 24], but a lower number of interruptions compared to interruptions on surgical wards and teams $[25,26]$. For example, in a study of operating room distractions, Antoniadis et al found that surgeons experienced interruptions 9.82 times per hour [27]. In a prior study in an ICU setting using smartphone technology found that sent and received messages totalled only 14.1 per day [28]. The relatively high numbers of interruptions seen in our study may be due to higher patient volumes or complexity at our sites or may be a reflection of the culture of communication at our sites. The higher number of interruptions seen on surgical wards may be due to the primary use of pagers in these studies for all communication including within the surgical team. At our institution, most communication between medical (rather than nursing) team members occurred through texting on personal smartphones and thus was not considered in the total communications, which likely lead to an underestimation of total interruptions.

A previous study has found that residents, staff physicians, and allied health members perceived that smartphones improved both efficiency and quality of care [29]. As well, centralizing communication to a team device is also used in academic centers to improve communication $[1,2]$. However, this change has made inter-professional communication easier and likely reduced the threshold to communicate, although rigorous data is lacking in this field due to lack of standardization definitions across studies. This has increased the total number of communications - and has led to several unintended negative consequences of improved information technology. Firstly, high volumes of interruptions are known to lead to medical errors and decreased efficiency in multiple health care dis- 
ciplines $[9,10,17,30,31]$. For example, in a study of diagnostic radiology residents, increased interruptions were associated with subsequently increased discrepant reports in the following hour [32]. Secondly, medical education is impacted due to the interruption of both formal and informal teaching sessions scheduled throughout the workday $[26,33,34]$. Additional negative impact of smartphone communications include reduced communicator accountability, inappropriate escalation of communication urgency, healthcare worker stress, and deterioration of interdisciplinary relationships $[23,35]$. Whereas pagers don't necessarily require urgent response because of cultural expectations, texts marked as urgent and certainly phone calls carry the inherent understanding of an immediate response. Therefore, these unintended negative consequences must be considered when evaluating the effect of transitioning from pagers to smartphones in health care settings.

Although centralization of communications to a single team smartphone can be burdensome, it does accomplish two important educational and training objectives in academic health centres. One, it allows senior residents carrying the device to learn how to delegate tasks and make prompt critical decisions. Secondly, junior residents are shielded against a flurry of interruptive communications and are therefore able to practice their clinical skills in their formative year. Thus, the high volume of communications and disruptions must be weighed against the benefits of centralized communication, which include training objectives for senior and junior residents.

In our study, we also introduced the new concept of excessive interruptions, termed 'crisis mode', and defined its quantitative threshold based on surveying of our residents and staff. Our results demonstrate that the frequency of the 'crisis mode' is unfortunately high during the workday, although it is unclear whether residents in our study eventually adapted to this level of disruption. Although defined elsewhere in a different context, our defined threshold for 'crisis mode' was based on a small sample size from our local institutions and therefore lacks in external validity and is based on the perceptions of our residents rather than a grounded operationalized definition [18]. However, as this definition previously does not exist in the literature, we have taken a first step in applying this novel concept to healthcare associated communications. Furthermore, this concept may be a future patient safety metric and therefore additional studies are needed to further validate this definition in similar settings and assess its impact on healthcare worker function and patient care.

There are several limitations to our study. First, both participating sites are part of the same university affiliated hospital system, which limits the study's external validity to academic centres using smartphones as their method of communication. Second, our study did not differentiate among the sources of interruptions nor content. Therefore, we were unable to assess the appropriateness of the interruptions. However, such a high volume of interruption especially during peak periods is likely unsafe regardless of indication. Third, we likely underestimated the total amount of interruptions experienced by residents as we did not include text messages and phone calls to personal communication devices, communication via personal pagers, and face to face interruptions. As well, we did not include outgoing communications from team devices, which, although these are no interruptions, are certainly consequences of interruptions that may increase the burden of work and disrupt flow.

Future directions of research need to examine how to reduce inappropriate interruptions rather than reducing the total volume of interruptions. Some studies have shown that one third of interruptions are in fact inappropriate in their urgency designation $[26,36]$. Potential interventions to reduce inappropriate interruption include inter-professional educational programs, decision support tools to assist health care staff in deciding appropriateness of communication, and utilizing electronic 'to do lists' to reduce interruptions of low-urgency communications. Research should also investigate the impact of technological interruptions on patient outcomes [37].

\section{Conclusion}

Our study demonstrated a high number of smartphone interruptions for residents working in CTUs, a metric correlating with patient volume and clinical site. Although direct patient and trainee impact was not assessed in our study, the high number of interruptions likely affected both patient care and trainee education. Future directions of investigation should investigate the sources of inappropriate interruptions as well as strategies to reduce their occurrence. 


\section{Clinical Relevance Statements}

There was a high level of smartphone interruptions for housestaff on the general internal medicine service.

The number of interruptions varied based on patient volume, site, and day of the week.

Physicians carrying the smartphones experienced frequent concentrated bursts of increased interruptions.

\section{Conflicts of Interest}

The authors declare that they have no conflicts of interest in the research.

\section{Human Subjects Protection}

The study was performed in compliance with the World Medical Association Declaration of Helsinki on Ethical Principles for Medical Research Involving Human Subjects, and was reviewed and approved by the University Health Network Institutional Review Board, Toronto, Ontario. 


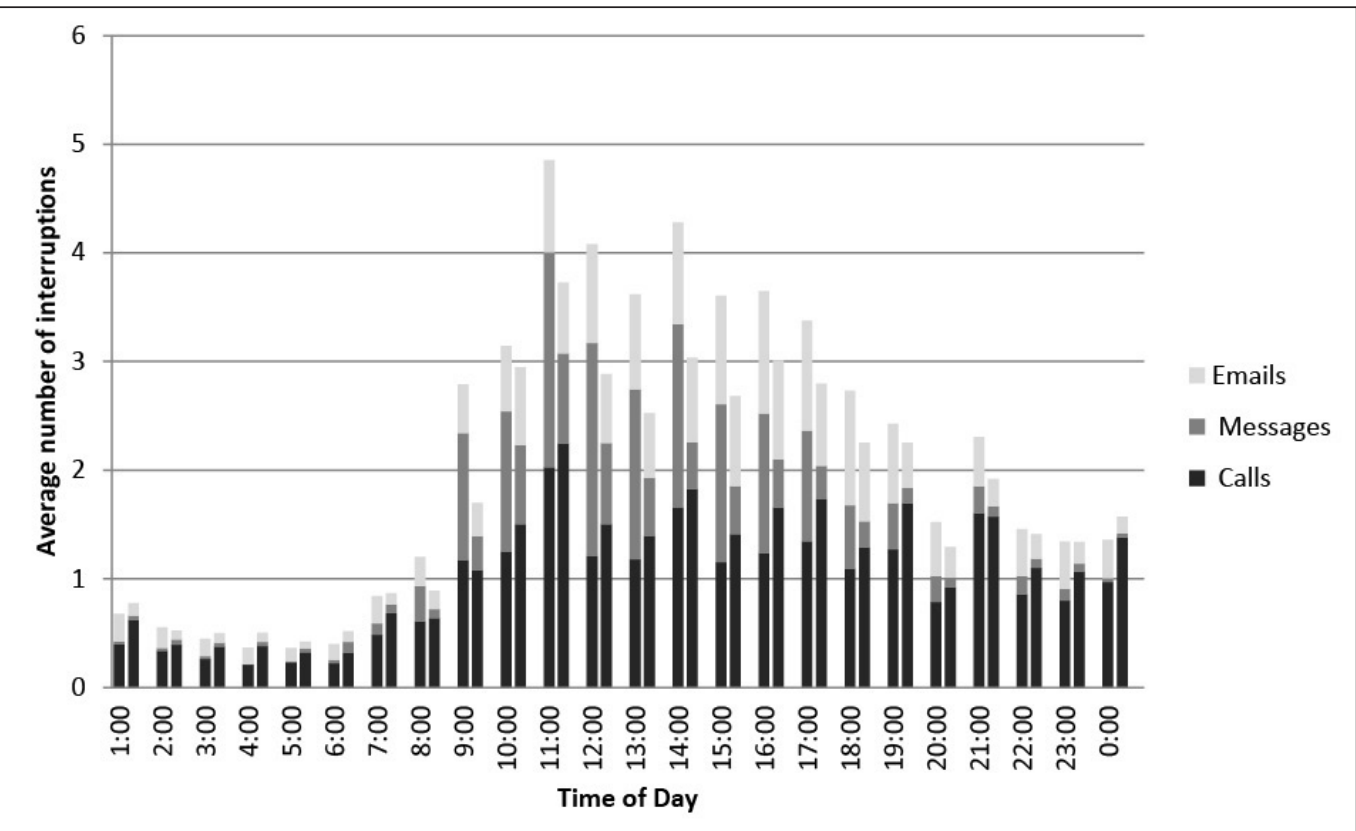

Fig. 1 Distribution of weekday interruptions received by residents carrying the team smartphone at each site (Column 1 -Toronto General Hospital, Column 2, Toronto Western Hospital)

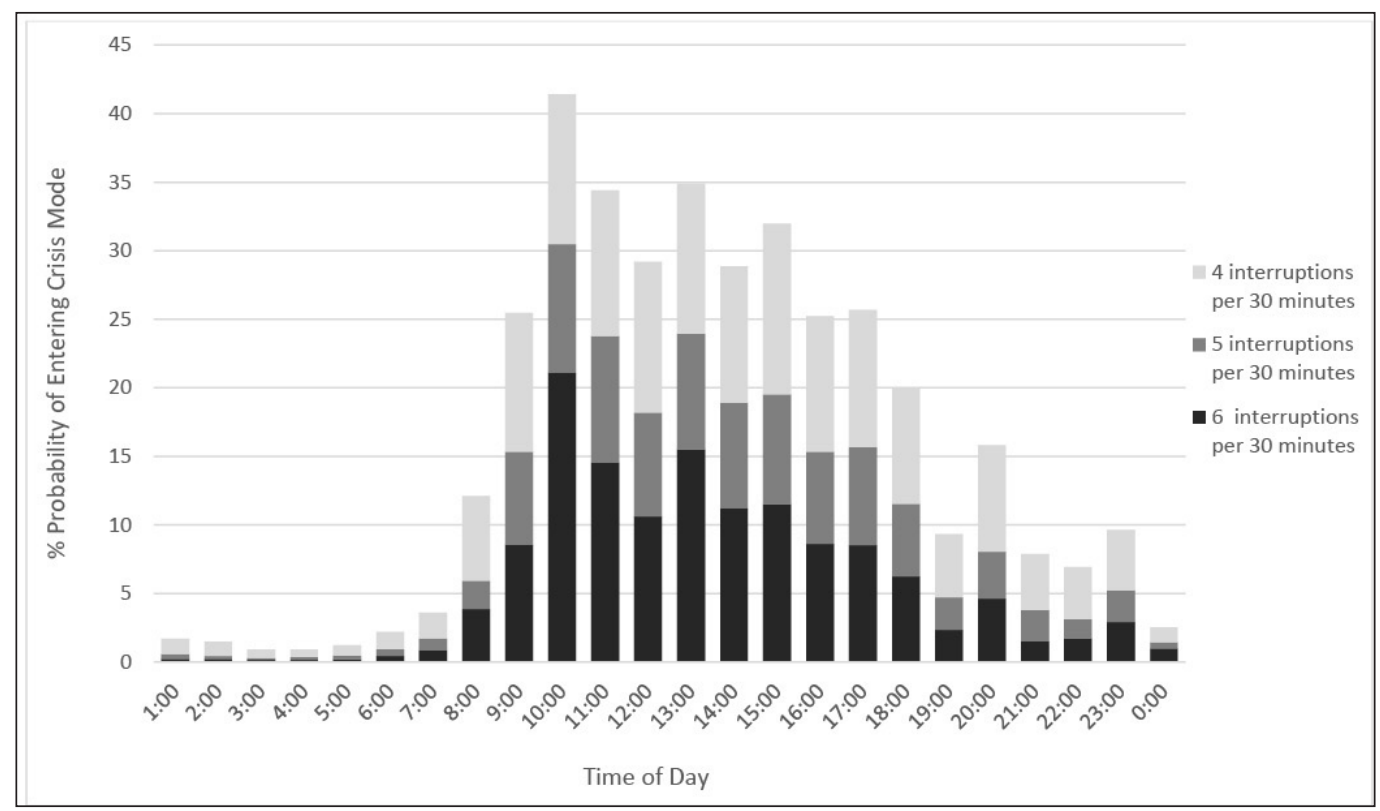

Fig. 2 Probability of entering 'crisis mode' by hour of day for a resident carrying a team smartphone, stratified by threshold of interruptions per hour 
Table 1 Interruptions by site and type of interruption, $\mathrm{N}=187049$

\begin{tabular}{|l|l|l|l|l|l|l|}
\hline & \multicolumn{2}{l}{ Site 1 } & \multicolumn{2}{l|}{ Site 2 } \\
\hline & $\begin{array}{l}\text { Daily Average } \\
\text { per team }\end{array}$ & $\begin{array}{l}\text { Total, all } \\
\text { teams }\end{array}$ & $\%$ & $\begin{array}{l}\text { Daily Average } \\
\text { per team }\end{array}$ & $\begin{array}{l}\text { Total, all } \\
\text { teams }\end{array}$ & $\%$ \\
\hline All Interruptions & 51.4 & 101279 & 54.1 & 42.4 & 85770 & 45.9 \\
\hline By Interruption Type & & & & & & \\
\hline Emails & 15.8 & 28283 & 27.9 & 6.0 & 10761 & 12.5 \\
\hline Text Messages & 13.3 & 26373 & 26.0 & 9.2 & 18422 & 21.5 \\
\hline Phones & 22.4 & 46623 & 46.0 & 27.1 & 56587 & 66.0 \\
\hline
\end{tabular}




\section{References}

1. Shieh L, Chi J, Kulik C, Momeni A, Shelton A, DePorte C, Hopkins J. Assigning a team-based pager for oncall physicians reduces paging errors in a large academic hospital. Jt Comm J Qual Patient Saf Jt Comm Resour 2014; 40(2): 77-82.

2. Wu RC, Lo V, Morra D, Wong BM, Sargeant R, Locke K, Cavalcanti R, Quan SD, Rossos P, Tran K, Cheung $M$. The intended and unintended consequences of communication systems on general internal medicine inpatient care delivery: a prospective observational case study of five teaching hospitals. J Am Med Inform Assoc JAMIA 2013; 20(4): 766-777.

3. Wong BM, Cheung CM, Dharamshi H, Dyal S, Kiss A, Morra D, Quan S, Sivjee K, Etchells EE. Getting the message: a quality improvement initiative to reduce pages sent to the wrong physician. BMJ Qual Saf 2012; 21(10): 855-862.

4. Mosa ASM, Yoo I, Sheets L. A systematic review of healthcare applications for smartphones. BMC Med Inform Decis Mak 2012; 12: 67.

5. Katz-Sidlow RJ, Ludwig A, Miller S, Sidlow R. Smartphone use during inpatient attending rounds: prevalence, patterns and potential for distraction. J Hosp Med 2012; 7(8): 595-599.

6. Przybylo JA, Wang A, Loftus P, Evans KH, Chu I, Shieh L. Smarter hospital communication: secure smartphone text messaging improves provider satisfaction and perception of efficacy, workflow. J Hosp Med 2014; 9(9): 573-578.

7. Wu RC, Morra D, Quan S, Lai S, Zanjani S, Abrams H, Rossos PG. The use of smartphones for clinical communication on internal medicine wards. J Hosp Med 2010; 5(9): 553-559.

8. Harvey R, Jarrett PG, Peltekian KM. Patterns of paging medical interns during night calls at two teaching hospitals. CMAJ Can Med Assoc J J Assoc Medicale Can 1994; 151(3): 307-311.

9. Flynn EA, Barker KN, Gibson JT, Pearson RE, Berger BA, Smith LA. Impact of interruptions and distractions on dispensing errors in an ambulatory care pharmacy. Am J Health-Syst Pharm AJHP Off J Am Soc Health-Syst Pharm 1999; 56(13): 1319-1325.

10. Drews FA. The frequency and impact of task interruptions in the ICU. Proc Hum Factors Ergon Soc Annu Meet 2007; 51(11): 683-686.

11. Potter P, Wolf L, Boxerman S, Grayson D, Sledge J, Dunagan C, Evanoff B. Understanding the cognitive work of nursing in the acute care environment. J Nurs Adm 2005; 35(7-8): 327-335.

12. Parker J, Coiera E. Improving clinical communication: a view from psychology. J Am Med Inform Assoc JAMIA 2000; 7(5): 453-461.

13. Weigl M, Müller A, Vincent C, Angerer P, Sevdalis N. The association of workflow interruptions and hospital doctors' workload: a prospective observational study. BMJ Qual Saf 2012; 21(5): 399-407.

14.Devlin MK, Kozij NK, Kiss A, Richardson L, Wong BM. Morning handover of on-call issues: opportunities for improvement. JAMA Intern Med 2014; 174(9): 1479-1485.

15.DeVita MA, Smith GB, Adam SK, Adams-Pizarro I, Buist M, Bellomo R, Bonello R, Cerchiari E, Farlow B, Goldsmith D, Haskell H, Hillman K, Howell M, Hravnak M, Hunt EA, Hvarfner A, Kellett J, Lighthall GK, Lippert A, Lippert FK, Mahroof R, Myers JS, Rosen M, Reynolds S, Rotondi A, Rubulotta F, Winters B. "Identifying the hospitalised patient in crisis"--a consensus conference on the afferent limb of rapid response systems. Resuscitation 2010; 81(4): 375-382.

16.Li SYW, Magrabi F, Coiera E. A systematic review of the psychological literature on interruption and its patient safety implications. J Am Med Inform Assoc JAMIA 2012; 19(1): 6-12.

17. Westbrook JI, Woods A, Rob MI, Dunsmuir WTM, Day RO. Association of interruptions with an increased risk and severity of medication administration errors. Arch Intern Med 2010; 170(8): 683-690.

18. Patterson ME, Bogart MS, Starr KR. Associations between perceived crisis mode work climate and poor information exchange within hospitals. J Hosp Med 2015; 10(3): 152-159.

19. Rivera-Rodriguez AJ, Karsh B-T. Interruptions and distractions in healthcare: review and reappraisal. Qual Saf Health Care 2010; 19(4): 304-312.

20. Coiera E, Tombs V. Communication behaviours in a hospital setting: an observational study. BMJ 1998; 316(7132): 673-676.

21. Shayne P, Heilpern K, Ander D, Palmer-Smith V, The Emory University Department of Emergency Medicine Education Committee. Protected Clinical Teaching Time and a Bedside Clinical Evaluation Instrument in an Emergency Medicine Training Program. Acad Emerg Med 2002; 9(11): 1342-1349.

22. Baethge A, Rigotti T, Roe RA. Just more of the same, or different? An integrative theoretical framework for the study of cumulative interruptions at work. Eur J Work Organ Psychol 2015; 24(2): 308-323.

23. Quan SD, Wu RC, Rossos PG, Arany T, Groe S, Morra D, Wong BM, Cavalcanti R, Coke W, Lau FY. It's not about pager replacement: an in-depth look at the interprofessional nature of communication in healthcare. J Hosp Med 2013; 8(3): 137-143. 
24. Katz MH, Schroeder SA. The sounds of the hospital. Paging patterns in three teaching hospitals. N Engl J Med 1988; 319(24): 1585-1589.

25. Patel SP, Lee JS, Ranney DN, Al-Holou SN, Frost CM, Harris ME, Lewin SA, Liu E, Madenci A, Majkrzak AA, Nelson J, Peterson SF, Serecky KA, Wilkinson DA, Wojcik BM, Englesbe MJ, Lynch RJ. Resident workload, pager communications, and quality of care. World J Surg 2010; 34(11): 2524-2529.

26. Fargen KM, O’Connor T, Raymond S, Sporrer JM, Friedman WA. An observational study of hospital paging practices and workflow interruption among on-call junior neurological surgery residents. J Grad Med Educ 2012; 4(4): 467-471.

27. Antoniadis S, Passauer-Baierl S, Baschnegger H, Weigl M. Identification and interference of intraoperative distractions and interruptions in operating rooms. J Surg Res 2014; 188(1): 21-29.

28. O'Connor C, Friedrich JO, Scales DC, Adhikari NKJ. The use of wireless e-mail to improve healthcare team communication. J Am Med Inform Assoc JAMIA 2009; 16(5): 705-713.

29. Wu R, Rossos P, Quan S, Reeves S, Lo V, Wong B, Cheung M, Morra D. An evaluation of the use of smartphones to communicate between clinicians: a mixed-methods study. J Med Internet Res 2011; 13(3): e59.

30. Sevdalis N, Undre S, McDermott J, Giddie J, Diner L, Smith G. Impact of intraoperative distractions on patient safety: a prospective descriptive study using validated instruments. World J Surg 2014; 38(4): $751-758$

31. Westbrook JI, Coiera E, Dunsmuir WTM, Brown BM, Kelk N, Paoloni R, Tran C. The impact of interruptions on clinical task completion. Qual Saf Health Care 2010; 19(4): 284-289.

32. Balint BJ, Steenburg SD, Lin H, Shen C, Steele JL, Gunderman RB. Do Telephone Call Interruptions Have an Impact on Radiology Resident Diagnostic Accuracy? Acad Radiol 2014; 21(12): 1623-1628.

33. Blum NJ, Lieu TA. Interrupted care. The effects of paging on pediatric resident activities. Am J Dis Child 1960 1992; 146(7): 806-808.

34. Wu RC, Tzanetos K, Morra D, Quan S, Lo V, Wong BM. Educational impact of using smartphones for clinical communication on general medicine: more global, less local. J Hosp Med 2013; 8(7): 365-372.

35. Klemets J, Evjemo TE. Technology-mediated awareness: Facilitating the handling of (un)wanted interruptions in a hospital setting. Int J Med Inf 2014; 83(9): 670-682.

36. Smith CNC, Quan SD, Morra D, Rossos PG, Khatibi H, Lo V, Wong H, Wu RC. Understanding interprofessional communication: a content analysis of email communications between doctors and nurses. Appl Clin Inform 2012; 3(1): 38-51.

37. Coiera E. The science of interruption. BMJ Qual Saf 2012; 21(5): 357-360. 